\title{
A comparative overview on good fats and bad fats: guide to control healthy body
}

\author{
Sujan Banik *, Mohammad Salim Hossain \\ Department of Pharmacy, Noakhali Science and Technology University, Sonapur, Noakhali-3814, Bangladesh \\ *Corresponding author E-mail: pharmasujan@yahoo.com
}

\begin{abstract}
An ideal food has six components such as carbohydrate, protein, fat, vitamin, salt and water. Every component is essential for our body for maintaining good health. Fat is necessary because it plays many critical roles in the body. It's a very rich source of energy that supports in cell function, helps insulate the body, protects us from injury, helps vitamin absorption and adds flavor and texture to foods. As well as fat also responsible for the risk of developing obesity, diabetes and heart disease in many cases. There are two classes of fat categorized in our listed daily food items; one is good fat (unsaturated fat) reduce the risk of developing heart diseases by helping to reduce blood cholesterol another is bad fat (saturated fat) can rise blood cholesterol. A fat diet, low in unsaturated fatty acids and rich in saturated fatty acids is thought to be an imperative basis of atherosclerosis and cardiovascular diseases. For maintaining good health, every person should update his knowledge regarding his food habits. This review focuses on the studies to summarize the good fats and bad fats from our daily listed food intake.
\end{abstract}

Keywords: Fat, Cholesterol, Saturated Fat, Trans Fat, Unsaturated Fat, Good Fat, Bad Fat.

\section{Introduction}

Fat is the most rigorous foundation of food energy. There are 9 calories in every gram of fats (Noakes, 2003). There are various group of compounds of this type of diet those are basically soluble in organic solvents and insoluble in water. Fat is defined chemically as triglycerides; trimesters of glycerol with several fatty acids (Brouwer et al., 2010). On the basis of their structure and composition fats may be both solid and liquid at room temperature. Several terms are frequently used to refer fats those are oils, fats and lipid, in which oils such as vegetable oil, canola oil, olive oil etc are liquids at normal room temperature, they are frequently used to refer fats, on the other hand fats is typically used to refer to fats that are solids at typical room temperature (e.g., lard, butter, shortening etc). Lipid is another familiar term which is generally worn to refer together liquid and solid fats, beside with other linked substances, generally in a medical and biochemical context. There are abundant diverse kinds of fats, but each is a distinction on the identical chemical arrangement. More specifically, the entire fats are sources of fatty acids and glycerol.

Cholesterol is a waxy like substance that is found in the fat cells of our body and in the bloodstream. Its function akin to as a "protective barrier' around the cells called a membrane. When the level of cholesterol is too elevated in our body, it can cause risk factors for other diseases to insist. There are largely two types of cholesterol; one is HDL (high density lipoproteins) and another is LDL (low density lipoproteins) cholesterol (Brouwer et al., 2010).

HDL cholesterol is also known as "good cholesterol" and LDL cholesterol is considered to be "bad cholesterol". Higher levels of HDL protects against heart attack and stroke because it keeps LDL cholesterol from building up around the heart. LDL cholesterol is carried through the blood stream and to the heart. When too much
LDL cholesterol builds up, it causes blockages in the big tubes (arteries) that carry blood to the heart. This damages the heart and over time can lead to a heart attack or stroke.

\section{Sources}

The main sources of fats are food items such as meat, egg yolk, milk, nuts, butter, ghee, cheese, different types of vegetables oils etc.

\section{Classification}

\subsection{Saturated fats}

It has no double bonds in the hydrocarbon chain and is solids at room temperature. Butter, ghee etc are familiar instance of saturated fats. High intakes of saturated fats help to raise blood cholesterol level (especially LDL cholesterol) that can guide to coronary disorders and high blood pressure (Ravnskov, 1998). The people who spend a sedentary daily life and old people have to reduce the ingestion of saturated fats. On the other hand, the energy requirement is high for the people who involved in heavy labor; they have to need fat intake more from their daily requirement.

\subsection{Unsaturated fats}

It has one or more double bonds and 'kink' in the hydrocarbon chain and is liquid at room temperature. Oils are unsaturated fats. Unsaturated fats may be monounsaturated fats, polyunsaturated fats (more than one double bond). High intake of these types of fats help to lower LDL (bad) cholesterol and help to raise HDL 
(good) cholesterol. Olives, Olive oil, sunflower oil, peanut oils are unsaturated fat.
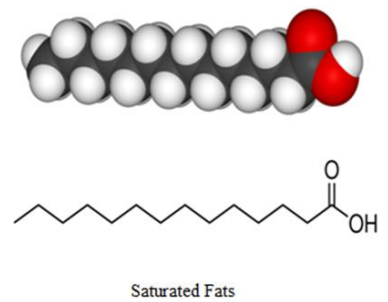

Fig. 1: Saturated and Unsaturated Fats

Depending on the dietary necessities, fats are two kinds:

a) Essential fats: Essential fats are categorized those types of fats that are not manufactured by the body such as linoleic acid, linolenic acid, arachidonic acid etc and must be included in the diet.

b) Non-essential fats: Those types of fats are synthesized by means of body and are not indispensable in the diet identified as non-essential fats. For instance: glycine.

\section{Functions of fats}

- Fats are the principal energy supplies of the body, which form a vital source of energy during hunger or other labor works.

- Fats take part in an important role in the absorption of fats soluble vitamins A, D, E and K.

- Control cholesterol level, so act as an important regulating compound.

- Help to maintain healthy skin and hair

- Acts like a "cushion" and heat regulator to protect your heart, liver and other vital organs

\section{Good fats, bad fats}

Therefore, from this study we can suggest that, all types of fats are not similar. Some of these are listed as essential for maintaining good health (good fat) and others listed as dangerous for our body (bad fat). The listed bad fats are responsible for increasing blood cholesterol levels or have also other harmful sound effects on cardiovascular health (Food Standard Board, 2007). After all, ingestion of excess fats of all categories can add intemperance calories and guide to weight gain. This review will help us to sort out the "heart healthy" (good) fats from the "unhealthy" (bad) fats.

\subsection{Heart healthy (good) fats}

Unsaturated fats are categorized as good fats. These types of fats are found chiefly in plant foods or in fish that eat tiny flora. Further unsaturated fats are categorized into two groups' monounsaturated fatty acid (MUFA) and polyunsaturated fatty acid (PUFA). The two polyunsaturated fatty acids crucial for physical condition are omega- 3 and omega- 6 . Omega- 3 and omega- 6 fatty are very essential for body because they play various superior role in our body but they cannot be synthesized by the body and be required to come from food. The beneficial effects of omega-3 fatty acids are brain and eye development of the growing fetus during pregnancy and for maintaining and promoting health all over life (Hooper, 2004). Omega-6 fatty acids also take part in development of brain and heart function and also in normal growth and development (Harris et al., 2009). Both mono-and polyunsaturated fats may help to lower LDL (bad) cholesterol and decrease risk of heart disease. Olive, canola and peanut oils are high in monounsaturated fats, and safflower, sunflower, and corn oils contain more polyunsaturated fats.

\subsubsection{Omega-3 fatty acids}

The common food sources of omega-3 fatty acids are fatty fish such as salmon, herring, sardines, trout and flaxseed, walnuts as well as canola oil. The American Heart Association (AHA) has suggested that healthy adults eat at least two servings of fish per week to boost omega-3 fatty acid intake (Lichtestein et al., 2006). It has numerous potential health promoting benefits, such as:

- Decrease inflammation in case of heart disease, inflammatory bowel disease and rheumatoid arthritis.

- Help prevent blood from clotting and sticking to artery walls.

- Reduce risk for blocked blood vessels and heart attacks.

- Prevent hardening of the arteries.

- Lower risk of sudden death and abnormal heart rates.

- Reduce triglyceride levels.

- Lower blood pressure.

\subsubsection{Omega-6 fatty acids}

The common sources of omega- 6 fatty acids are vegetable oils such as corn oil, safflower oil, sesame oil, soybean oil, sunflower oil; soft (liquid or tub) margarine, preferably one that is trans-fatfree; walnuts; sunflower seeds, pumpkin seeds, sesame seeds; soy "nuts" (roasted soy beans), soy nut butter and tofu etc.

Omega-6 fatty acids also play key roles in health promoting benefits, for instance:

- Neutral or reduce levels of inflammatory mediators.

- Replacing saturated and trans fat with omega-6 fatty acids linked with decreasing risk of heart disease.

- Develop insulin resistance and decrease the occurrence of diabetes

- Inferior blood pressure.

- Inferior cholesterol levels.

\subsubsection{Monounsaturated fats}

The available food sources of monounsaturated fats are vegetable oils: olive oil, canola oil, peanut oil; nuts: almonds, cashews, peanuts, pecans, pistachios; avocado; peanut butter and almond butter.

The main functions of this lower LDL cholesterol, while increasing HDL (good) cholesterol.

\subsection{Unhealthy fats (bad fats)}

Those types of fats shows unwanted effects in our body are known as bad fats such as saturated fats and Trans fats (Uauy et al., 2009; Jakobsen, 2008). Meats with high-fat and dairy foods such as cheese, whole milk etc is the main foundation of saturated fatty acid. The enlisted food items are the most important resource of unhealthful fats and have to avoid or eaten scarcely.

\subsubsection{Saturated fats}

The familiar food sources of saturate fats are fatty cuts of beef, pork and lamb; poultry skin, chicken wings, dark meat chicken; high fat dairy products: cheese, butter, whole milk, $2 \%$ reduced fat milk, cream, cream cheese, sour cream, ice cream; tropical oils: coconut oil, palm oil, palm kernel oil, cocoa butter; lard and suet etc.

\subsubsection{Tran's fatty acids (Tran's fats)}

Tran's fats (occasionally called Trans fatty acids) are produced during the progression of hydrogenation of vegetable oils (Mozaffarin et al., 2006; Bakker et al., 1997). Hydrogenation is a method used by food manufacturers to add hydrogen to vegetable oils to create a solid fat. Tran's fat is the worst kind of fat, even worse than saturated fat. But when shopping for food, always check labels and choose products with 0 grams of Tran's fat. 
Trans fat also known as partially hydrogenated oil. It's called hidden killer of our body.

Food Sources: There are two sources of Tran's fat, one is natural and another is manmade. The common sources of Tran's fat such as vegetable shortening and stick margarines; refrigerated dough products; fried foods: doughnuts, French fries, other deep fried fast food items; commercially ready foods containing partially hydrogenated oils: crackers, cookies, cakes, pastries, microwave popcorn and other snack foods.
High intake of saturated fats and Trans fats can raise the level of bad cholesterol (LDL cholesterol) in our body. These fats may also make the lining of blood vessels (the endothelium) less flexible. In adding together, Tran's fats may demoralize the "good" blood cholesterol (HDL cholesterol) level when eaten in large quantities. Tran's fats can have an effect on our health - especially our heart health.

From the basis of 'Good Fats' and 'Bad Fats' we can classify fats by the following ways: 3

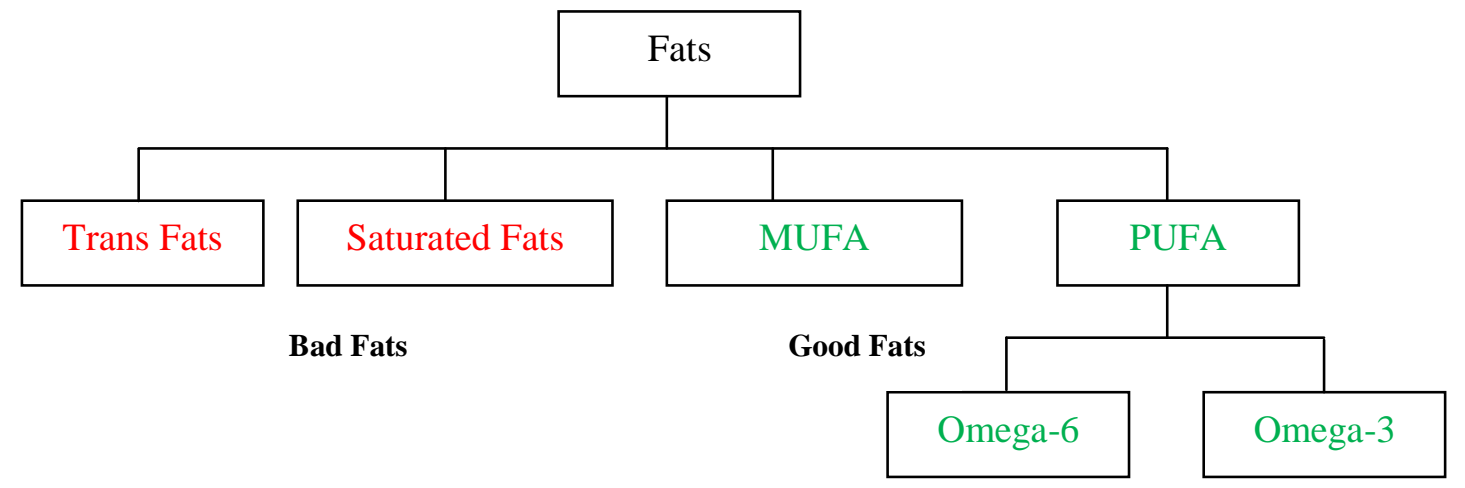

Table 1: Guide to Control Healthy Body Choose the Food Items in This Order

\begin{tabular}{ccc} 
Avoid & Eat less & Eat \\
Trans Fat & Good Fats \\
\hline & & \\
\hline
\end{tabular}

\section{Daily restrictions for fat ingestion}

According to the U.S. Department of Agriculture and the Department of Health and Human Services (HHS) recommendation the daily limit of fat intake should not more 35 percent of your daily calories (Ward, 2010). This recommendation notice that, if any person consume 1800 calories a day, then he/she have to eat no more than 70 grams of fat daily (To calculate: $1,800 \times 0.35=630$ calories, $630 / 9=70 \mathrm{~g}$ total fat). This calculation is an upper limit of fat intake and the majority of these fat calories appear from monounsaturated and polyunsaturated sources. By the organization of USDA and NHS at yet not established an upper limit for tans fat, but they recommended that your trans-fat intake should be low as possible. According to American Heart Association the set value of upper limit of Trans fat intake not more than 1 percent of your daily calories (Kaneshiro, 2009). Once you can choose a product contrast nutrition facts labels that are lowest in saturated fat, Trans fat and cholesterol. Always choose the product with the lowest amount of saturated fat, 0 grams tans fat and with less cholesterol (Mozaffarin et al., 2006).

\section{Conclusion}

There is a widespread false impression that all body fat is not good. In reality, a number of body fats are essential to guard the person's health as well as supplying a pool of energy for performing a variety of body functions. To know better this thought, the total weight of body fat can be further divided into three divide categories: 
- The amount of body fat that is essential to keep the body from any type of infectious diseases and to protect the internal organ from bruising damage is known to be Essential Body Fat.

- The amount of additional body fat that does not root of any medical risks, and provides a pool of "fuel" for employ by the body is known to be Reserve Body Fat.

Another is Excess Body Fat, which is above and over the grouping of essential body fat together with reserve body fat. It causes the menace of severe health problem such as strokes, heart attacks, diabetes and certain forms of cancer.

\section{References}

[1] Bakker N, van't Veer P \& Zock PL (1997) Adipose fatty acids and cancers of the breast, prostate and colon: and ecological study. International Journal of Cancer 72, 587-91. http://dx.doi.org/10.1002/ (SICI) 1097-0215(19970807)72:4<587: AID-IJC6>3.0.CO; 2-M.

[2] Brouwer IA, Wanders JA \& Katan MB (2010) Effect of animal and industrial Tran's fatty acids on HDL and LDL cholesterol levels in humans A Quantitative review. PLoS One 5, E9434. http://dx.doi.org/10.1371/journal.pone.0009434.

[3] Food Standards Agency Review Board (2007) Agency board paper: Trans fatty acids.

[4] Good Fats/Bad Fats, How to know the difference. Health bulletin New York City Department of Health and Mental Hygiene, volume 9, number 1.

[5] Harris WS, Appel LJ \& Engler MM (2009) Omega-6 Fatty Acids and Risk for Cardiovascular Disease: A Science Advisory from the American Heart Association Nutrition Subcommittee of the Council on $\mathrm{Nu}$ trition, Physical Activity, and Metabolism. 119, 902-907.

[6] Hooper L (2004) Omega 3 fatty acids for prevention and treatment of cardiovascular disease. Cochrane database. Systemic Review Oct. 18(4).

[7] Jakobsen M (2008) Intake of ruminant Tran's fatty acids and risk of coronary heart disease. International Journal of Epidemiology 37, 173 82. http://dx.doi.org/10.1093/ije/dym243.

[8] Kaneshiro NK. (2009, Aug. 2). In Fat: MedlinePlus Medical Encyclopedia. Retrieved June 1, 2010, from http://www.nlm.nih.gov/medlineplus/ency/article/002468.htm.

[9] Lichtestein A, Appel L \& Brands M (2006) Diet and Lifestyle Recommendations Revision 2006: A Scientific Statement from American Heart Association Nutrition Committee. 114: 82-96.

[10]Mozaffarian D, Katan M \& Ascherio A (2006) Trans fatty acids and cardiovascular disease. New England of Medicine 354 (13), 16011613. http://dx.doi.org/10.1056/NEJMra054035.

[11]Noakes T. 2003. Lore of Running. Oxford University Press: Human Kinetics.

[12]Ravnskov U (1998) the Questionable Role of Saturated and Polyunsaturated Fatty Acids in Cardiovascular Disease. Journal of Clinical Epidemiology 51(6), 443-460. http://dx.doi.org/10.1016/S0895-4356 (98)00018-3

[13]Uauy R, Aro a \& Clarke R (2009) WHO Scientific Update on Trans fatty acids: summary and conclusions. European Journal of Clinical Nutrition 63, S68-S75. http://dx.doi.org/10.1038/ejcn.2009.15.

[14]Ward EM. In Fat Facts: Good Fats vs. Bad Fats. 2008. Retrieved June 1, 2010, from http://www.webmd.com/food-recipes/features/goodfats-bad-fats. 